\title{
The influence of intruder states in even-even Po isotopes
}

\author{
J.E. García-Ramos ${ }^{1, a)}$ and K. Heyde ${ }^{2, b)}$ \\ ${ }^{1}$ Departamento de Física Aplicada, Universidad de Huelva, 21071 Huelva, Spain \\ ${ }^{2}$ Department of Physics and Astronomy, Ghent University, Proeftuinstraat 86, B-9000 Gent, Belgium \\ a)enrique.ramos@dfaie.uhu.es \\ b) kris.heyde@ugent.be
}

\begin{abstract}
We study the role of intruder states and shape coexistence in the even-even ${ }^{190-206}$ Po isotopes, through an interacting boson model with configuration mixing calculation. We analyzed the results in the light of known systematics on various observable in the $\mathrm{Pb}$ region, paying special attention to the unperturbed energy systematics and quadrupole deformation. We find that shape coexistence in the Po isotopes behaves in very much the same way as in the Pt isotopes, i.e., it is somehow hidden, contrary to the situation in the $\mathrm{Pb}$ and the $\mathrm{Hg}$ isotopes.
\end{abstract}

\section{INTRODUCTION}

Shape coexistence has been observed in many mass regions throughout the nuclear chart and has become a very useful paradigm to explain the competition between the monopole part of the nuclear effective force that tends to stabilize the nucleus into a spherical shape, in and near to shell closures, and the strong correlations (pairing, quadrupole in particular) that favors the nucleus into a deformed shapes in around mid-shell regions [1].

New experimental results pushed our knowledge on the even-even $\mathrm{Pt}, \mathrm{Hg}, \mathrm{Pb}$, and $\mathrm{Po}$ isotopes towards region far from the region of stable nuclei, for which experimental information was lacking and highly needed. In this mass region the intruder bands are easily singled out for the $\mathrm{Pb}$ and $\mathrm{Hg}$ isotopes and the excitation energies display the characteristic parabolic pattern with minimal excitation energy around the $\mathrm{N}=104$ neutron mid-shell nucleus. However, this structure seems absent in the Pt and Po isotopes. Indeed, in the case of Pt, instead of exhibiting a parabolic shape in the energy systematics, there appears a sudden drop in the excitation energies for certain states: $0_{2}^{+}$, $4_{1}^{+}, 2_{3}^{+}$and $6_{1}^{+}$, followed by a flat behavior around midshell. In the case of Po, it is even more difficult to disentangle the interplay between regular and intruder states because the detailed spectroscopic data is not available going below neutron number $\mathrm{N}=106(\mathrm{~A}=190)$, i.e., the mid-shell nucleus is not reached experimentally. In particular, the energy of the $2_{1}^{+}$state remains rather constant $(\sim 600-650 \mathrm{keV})$ in the mass range $\mathrm{A}=196-208$, with a sudden drop at $\mathrm{A}=196$, with corresponding energy of $\sim 300 \mathrm{keV}$.

In a series of articles, we studied the Pt isotopes extensively [2], as well as the $\mathrm{Hg}$ isotopes [3], with the Interacting Boson Model (IBM) [4] incorporating proton 2p-2h excitations (IBM-CM) [5]. The conclusion of these studies was that configuration mixing in the Pt isotopes is somehow "concealed", while in the case of $\mathrm{Hg}$ isotopes (as well as for $\mathrm{Pb}$ nuclei) the presence of intruder states is self-evident.

The IBM-CM allows the simultaneous treatment and mixing of several boson configurations corresponding to different particle-hole shell-model excitations [5]. Hence, the model space corresponds to a $[N] \oplus[N+2]$ boson space. The boson number $N$ is obtained as the sum of the number of active protons (counting both proton particles and holes) and the number of valence neutrons, divided by two. Thus, the Hamiltonian for two-configuration mixing is written

$$
\hat{H}=\hat{P}_{N}^{\dagger} \hat{H}^{N} \hat{P}_{N}+\hat{P}_{N+2}^{\dagger}\left(\hat{H}^{N+2}+\Delta^{N+2}\right) \hat{P}_{N+2}+\hat{V}_{\operatorname{mix}}^{N, N+2},
$$

where $\hat{P}_{N}$ and $\hat{P}_{N+2}$ are projection operators, $\hat{V}_{\text {mix }}^{N, N+2}$ describes the mixing both subspaces, $\hat{H}^{i}$ is the IBM Hamiltonian with $i=N, N+2$, and $\Delta^{N+2}$ is related to the energy needed to excite two particles across the shell gap.

Within this formalism we have performed a fit to the excitation energies and $B(E 2)$ transition rates of ${ }^{190-208}$ Po in order to determine the parameters for the IBM-CM Hamiltonian. The results from the fitting procedure are summarized 
in Table II of Ref. [6] and are used in the calculations and results discussed in the present contribution. Here, we present IBM-CM calculations for the Po isotopes, focusing on excitation energies as well as on the quadrupole deformation, and, compare the corresponding results in the $\mathrm{Pt}$ and $\mathrm{Hg}$ isotopic series.

\section{ABSOLUTE ENERGIES AND ENERGY SYSTEMATICS}

Intruder states are expected to appear, in principle, at an excitation energy well above the energy of the corresponding regular ones. Therefore, at a first glance, the ground state would always be of regular nature. The reason is that intruder configurations are related to the creation of $2 \mathrm{p}-2 \mathrm{~h}$ excitations across the $\mathrm{Z}=82$ closed shell. These energies correspond to $\Delta^{N+2}=3480 \mathrm{keV}$ [3], $2400 \mathrm{keV}$ [6], and $2800 \mathrm{keV}$ [2], for the $\mathrm{Hg}$, Po, and Pt isotopes, respectively. However, this energy gap has to be corrected by the pairing and quadrupole energy gain that can reduce notably this energy difference. In particular, around the mid-shell point at $\mathrm{N}=104$ where the number of active nucleons becomes maximal, the energy gain due to the strong correlation energy is such that the energy of the intruder configurations becomes close, or even crosses the energy of the regular ones. On top of this, there is the mixing between both configurations, that can become large, making it difficult, in some cases, to determine which configurations are dominant in the ground-state wavefunction.

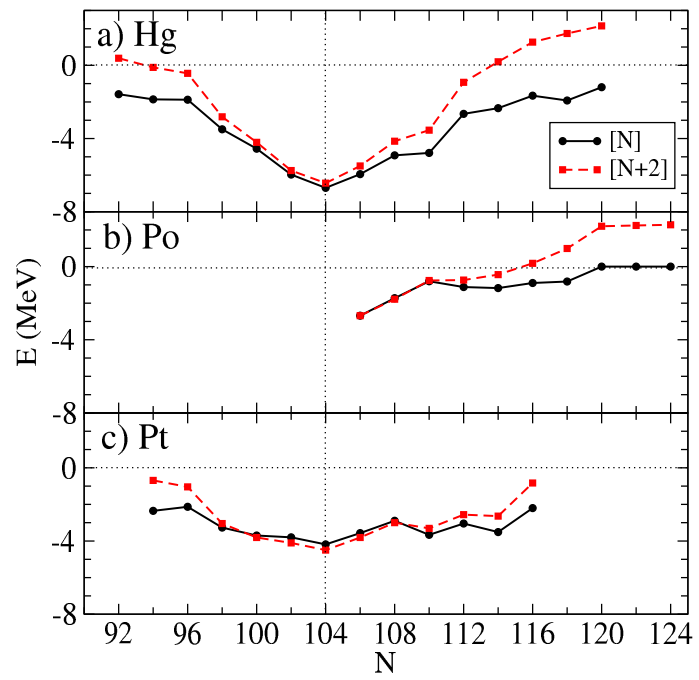

FIGURE 1. Absolute energy of the lowest regular [N] and intruder states [N+2] for ${ }^{172-200} \mathrm{Hg}$ (panel a), ${ }^{190-208} \mathrm{Po}($ panel b), and ${ }^{172-194} \mathrm{Pt}$ (panel c). The same energy scales are used for the three panels.

To face this problem, we calculate the "absolute" energy of the lowest $0^{+}$state belonging to both the $[\mathrm{N}]$ regular and $[\mathrm{N}+2]$ intruder configuration spaces, turning off the interaction amongst the two families $\left(\hat{V}_{\operatorname{mix}}^{N, N+2}=0\right)$. The energy of the lowest $0^{+}$state in the regular configuration space $[\mathrm{N}]$ and in the intruder configuration space $[\mathrm{N}+2]$ is lowered by their specific correlation energy. In general the energy reduction is larger for the intruder configuration as compared with the regular configuration, mainly because the number of bosons is larger. Therefore, the nature of the ground state will depend on the detailed balance between the value of $\Delta^{N+2}$ and the correlation energies. In Fig.1 we compare the unperturbed (with no mixing) first regular and intruder $0^{+}$states, for $\mathrm{Hg}$ (panel a), Po (panel b), and Pt (panel c). First, one notices that for the $\mathrm{Hg}$ isotopes, the lowest configuration is always the regular one, even at midshell. On the other hand, for Pt isotopes, in most cases the regular configuration describes the ground state, except around midshell, where the intruder configuration takes over to describe the ground state. Finally, one appreciates for the Po isotopes, a strong similarity with the Pt isotopes, with the intruder configuration becoming the ground state around midshell. There is a difference though, because in the Po isotopes both configurations are almost degenerate in energy while in the Pt isotopes, the intruder configurations appear well below the regular configurations around midshell. A full calculation with the analysis of the wave functions confirms the above conclusions (see Refs. [2, 3, 6]).

Regarding the experimental energy systematics of the intruder states, one expects a parabolic shape centered around $\mathrm{N}=104$. However, this shape can be strongly perturbed because of the mixing between regular and intruder 


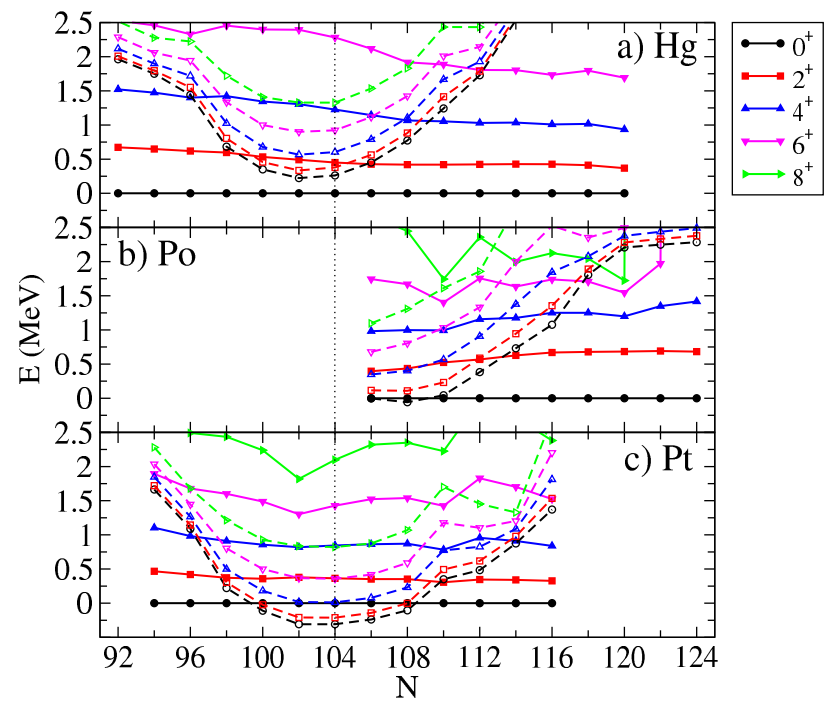

FIGURE 2. Comparison of the energy spectra for the IBM-CM Hamiltonian, switching off the mixing term. The two lowest-lying regular states and the lowest-lying intruder state for each of the angular momenta are shown (full lines with closed symbols for the regular states while dashed lines with open symbols are used for the intruder ones).

configurations and because of the crossing in energy of these two configurations, implying a change in the groundstate wavefunction. To obtain better insight into the energy systematics of intruder and regular states, it is enlightening to calculate the energy spectra as a function of $\mathrm{N}$ switching off the mixing part of the Hamiltonian. In Fig. 2 we depict the spectra, separating regular and intruder states, for the $\mathrm{Hg}$ (panel a), Po (panel b), and Pt (panel c) isotopes. Comparing the three isotopic series, one can appreciate a common picture that cannot be observed easily inspecting the experimental energy spectra (except in the case of $\mathrm{Hg}$ due to its small mixing term). In fact, in the three isotope series, the intruder configurations indeed present the expected parabolic shape, while the regular ones exhibit a rather flat energy behavior. The only, but important, difference between the three panels is the relative position in energy of the intruder and regular states. Note that for the Po and Pt isotopes, it is not possible to clearly see, from the experimental data solely, a different pattern in the energy systematics of regular and intruders because of the strong mixing and due to the crossing of regular and intruder configurations in the ground state around midshell.

\section{NUCLEAR DEFORMATION}

In this section, we use a phenomenological approach to extract information on the quadrupole deformation $\beta$ for both the regular and the intruder configurations. To this end we rely on the geometrical picture of the nucleus in order to extract the $\beta$ parameter for a given band, either regular or intruder, from known $B(E 2)$ values

$$
\beta=\frac{4 \pi \sqrt{B\left(E 2: I_{i} \rightarrow I_{f}\right)}}{3\left\langle I_{i} K 20 \mid I_{f} 0\right\rangle Z e R_{0}^{2}},
$$

where $R_{0}=1.2 A^{1 / 3} \mathrm{fm}$ and $<\ldots . . . .>$ is the Clebsch-Gordan coefficient. The key point is the assumption that around midshell, the $0_{1}^{+}$and $2_{1}^{+}$states belong to the regular (or intruder) band while the $6_{1}^{+}$and $4_{1}^{+}$states belong to the intruder (or regular) band. Therefore, using the corresponding E2 transitions, one can extract in a separate way the deformation corresponding with the intruder and regular configurations. When removed far from midshell, most probably both transitions will involve regular states because in this region intruder are supposed to be well above in energy (see also Fig. 2).

In Fig. 3, we depict the value of $\beta$ using the above method for the $\mathrm{Hg}$ (panel a), the Po (panel b), and the Pt (panel c) isotopes. In the case of $\mathrm{Hg}$, one easily singles out the presence of two configurations with very different deformation; the lower configuration, which corresponds to the regular state, is less deformed than the higher one and can be identified with an intruder state. This is an empirical evidence about the nature of the ground state which, close 


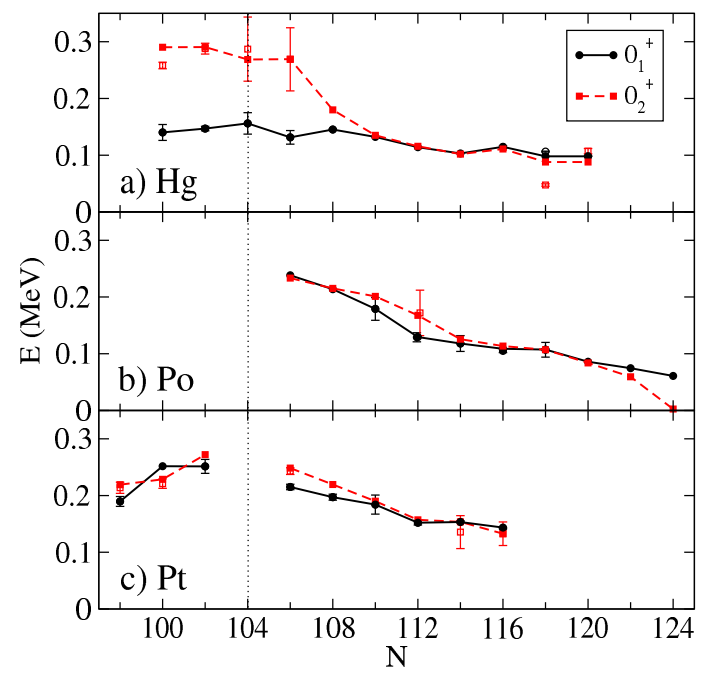

FIGURE 3. Comparison of the value of $\beta$ extracted from experimental and theoretical $\mathrm{B}(\mathrm{E} 2)$ values corresponding to the $0_{1}^{+}$and $0_{2}^{+}$states.

to midshell, always corresponds to a less deformed and regular configuration. In the Po and Pt isotopes, a completely different systematics compared with $\mathrm{Hg}$ shows up. Both configurations present a rather similar deformation, which is a consequence of the large mixing between both families of configurations and therefore it is not possible to discriminate them. The maximum deformation is reached around midshell where a bump is observed.

\section{CONCLUSIONS}

In this contribution, we have, starting from new IBM-CM calculations performed for the Po isotopes [6], compared those results with previous results obtained for the $\mathrm{Hg}$ and $\mathrm{Pt}$ isotopes using the same approach. Our main conclusion is the strong similarity between the Po and Pt isotopes as well as striking differences with the Hg isotopes (as well as with the $\mathrm{Pb}$ isotopes). In the Po isotopes, strong mixing between the regular and intruder configurations results from the calculations, with the intruder configuration becoming dominant in describing the $0^{+}$ground state wavefunction around midshell. In summary, configuration mixing is concealed in the Po isotopes, very much like it is the case for the Pt isotopes, while in $\mathrm{Hg}$, the presence of the intruder configurations has become self-evident.

\section{ACKNOWLEDGMENT}

Financial support from the "FWO-Vlaanderen" (KH and JEGR) and the InterUniversity Attraction Poles Programme - Belgian State - Federal Office for Scientific, Technical and Cultural Affairs (IAP Grant No. P7/12, is acknowledged. This work has also been partially supported by the Spanish MINECO and the FEDER under Project No. FIS201128738-C02-02 and by Spanish Consolider-Ingenio 2010 (CPANCSD2007-00042).

\section{REFERENCES}

[1] K. Heyde and J.L. Wood, Rev. Mod. Phys. 83, 1467 (2011).

[2] J.E. García-Ramos and K. Heyde, Nucl. Phys. A 825, 39 (2009); J.E. García-Ramos, V. Hellemans, and K. Heyde, Phys. Rev. C 84, 014331 (2011);

[3] J.E. García-Ramos, and K. Heyde, Phys. Rev. C 89, 014306 (2014); J.E. García-Ramos, and K. Heyde, EPJ Web of Conferences 93, 01004 (2015).

[4] F. Iachello and A. Arima, The Interacting Boson Model, Cambridge University Press (1987).

[5] P.D. Duval and B.R. Barrett, Nucl. Phys. A 376, 213 (1982).

[6] J.E. García-Ramos and K. Heyde, to appear in Phys. Rev. C. 